\title{
Influencia de la relación velocidad de corte-avance en la calidad superficial de muestras taladradas de fibra de carbono
}

\author{
M. Álvarez*, M.S. Carrilero*, J.E. Ares**, J.M. González* y M. Marcos* \\ Resumen En los últimos años, la fibra de carbono (FC) encuentra, cada vez, más aplicaciones en los \\ distintos sectores industriales. En el caso particular de la industria aeronáutica, este tipo de \\ materiales se somete frecuentemente a operaciones de taladrado formando parte de \\ estructuras híbridas FC/substrato metálico, debido a las necesidades de montaje en los \\ elementos estructurales de una aeronave. Sin embargo, en el taladrado de FC se puede \\ presentar una serie de problemas como las delaminaciones, la pérdida de calidad en el \\ acabado superficial o el desgaste rápido y excesivo de la herramienta de corte. En este \\ trabajo se lleva a cabo un primer estudio de la calidad de acabado superficial obtenida en \\ muestras de FC taladradas en función de las condiciones de corte impuestas.
}

Palabras clave Fibra de carbono. Taladrado. Calidad superficial. Delaminación. v/a

\section{Influence of the cutting speed-feed relation on the surface quality of drilled carbon fiber samples}

\begin{abstract}
The industrial applications of Carbon Fiber (CF) have increased in the last years. Particularly, in the aerospace industry, these materials are usually drilled because of the assembly requirements of the structural elements of airships. However, drilling of CF can be accompanied of troubles such as delaminations, lost of surface quality and quick wear of cutting tool. In this work, a study on the surface quality of CF drilled samples as a function of the cutting parameters has been achieved.
\end{abstract}

Keywords

Carbon Fiber. Drilling. Surface quality. Delamination. v/a

\section{INTRODUCCIÓN}

El desarrollo de la industria aeroespacial va íntimamente ligado al diseño de nuevos materiales cada vez más ligeros y con mejores propiedades mecánicas. Así, en los últimos quince años, la elevada resistencia mecánica específica y la excelente relación peso/propiedades mecánicas de la fibra de carbono y de los materiales compuestos de matriz metálica (MMC) han promovido su creciente aplicación en la construcción aeronáutica. Adicionalmente, la investigación en este tipo de materiales ha supuesto un notable descenso en el coste de su proceso de fabricación, lo que, junto con las causas anteriormente referidas, los ha convertido en materiales especialmente atractivos a escala industrial $^{[1 \text { y } 2]}$.
El continuo interés por todos estos materiales ha permitido el desarrollo de distintos procesos de mecanizado no convencionales, como el corte por láser o el corte por chorro de agua, para el procesado de los mismos ${ }^{[3 \text { y }}{ }^{4]}$. No obstante, siguen usándose ampliamente otros procesos convencionales como el fresado y, especialmente, el taladrado. En la industria aeronáutica, este último proceso es el empleado con más frecuencia debido a las necesidades de montaje en los elementos estructurales de una aeronave ${ }^{[3]}$. Sin embargo, en el taladrado de FC se puede presentar una serie de problemas como las delaminaciones, la pérdida de calidad en el acabado superficial o el desgaste rápido y excesivo de la herramienta de corte ${ }^{[1 \text { y } 5-8]}$.

En este trabajo se lleva a cabo un estudio de la calidad de acabado superficial obtenida en

(*) Departamento de Ingeniería Mecánica y Diseño Industrial. Universidad de Cádiz. Escuela Superior de Ingeniería. c/ Chile s/n, E-11003, Cádiz, SPAIN. Teléfono.+34 956.015123. Fax.+34 956.015101.E-mail: mariano.marcos@uca.es

(**) Departamento de Diseño de la Ingeniería. Universidad de Vigo. Escuela Técnica Superior de Ingenieros Industriales y de Minas. LagoasMarcosende, E-36200, Vigo (Pontevedra), SPAIN. 
muestras taladradas con distintos materiales de herramienta en función de los parámetros de corte aplicados.

\section{MATERIALES Y PROCEDIMIENTO EXPERIMEN- TAL}

El estudio realizado se ha llevado a cabo sobre placas de fibra de carbono de $16 \mathrm{~mm}$ de espesor, con entrelazado a 45 y $90^{\circ}$ y con curado en autoclave. Los ensayos se realizaron en un Centro de Mecanizado de Emco, modelo VMC-300 equipado con control numérico Siemens. Para los ensayos se aplicaron velocidades entre 700 y 3.000 r.p.m. y avances entre 0,05 y $0,3 \mathrm{~mm} / \mathrm{rev}$. La profundidad de corte no ha sido objeto de estudio en esta fase y su influencia se analizará en posteriores trabajos. Con objeto de analizar las condiciones de máxima agresividad, las placas se mecanizaron en seco.

Los procesos de taladrado se llevaron a cabo empleando herramientas de metal duro (WC-Co) sin recubrir y con recubrimientos de $\mathrm{TiN}$ y CrN, y herramientas sin recubrir de acero rápido (HSS). Estas herramientas fueron sometidas a inspección visual tras periodos de $1,2,3$, y 5 taladros, dependiendo de la respuesta al corte de las mismas. Dicha inspección se llevó a cabo con una cámara digital Nikon Coolpix 4500.

La calidad superficial de los taladros se midió en términos de la rugosidad superficial de los interiores y de la delaminación en los orificios de entrada y salida, en función de la relación entre la velocidad de corte y el avance, v/a. Para la medida de la rugosidad se empleó un rugosímetro Perthen, especialmente equipado y adaptado para ese tipo de medidas. Como parámetro de medida se tomó la rugosidad media, $\mathrm{R}_{\mathrm{a}}$. Por su parte, la delaminación se determinó a partir del denominado Factor de Delaminación, $\mathrm{D}_{\mathrm{L}}$, definido por ${ }^{[1]}$ :

$$
\mathrm{D}_{\mathrm{L}}=\mathrm{D}_{\mathrm{M}} / \mathrm{D}_{0}
$$

En esta expresión, $D_{M}$ es el diámetro máximo de la zona dañada y $\mathrm{D}_{0}$ el diámetro nominal del orificio y depende directamente de la relación $\mathrm{v} / \mathrm{a}^{[7]}$.

Finalmente, se ha establecido un índice de calidad $^{[9]}$, QI, definido por:

$$
\mathrm{QI}=\mathrm{K}_{\mathrm{I}} \cdot\left(\mathrm{R}_{\mathrm{a}} \cdot \mathrm{D}_{\mathrm{L}}\right)^{-1} \cdot \mathrm{N}_{\mathrm{T}}
$$

En la ecuación (2), $\mathrm{K}$ depende de la relación v/a y $\mathrm{N}_{\mathrm{T}}$ es el número de taladros antes de la rotura de la broca.

\section{RESULTADOS Y DISCUSIÓN}

Como se ha comentado previamente, uno de las principales causas de la pérdida de calidad superficial en los taladros se debe a la delaminación producida en la fibra de carbono en los puntos de entrada y salida de la herramienta. En la figura 1 se muestran los efectos de delaminación producidos por la entrada de la herramienta (a) y por la salida de la misma (b). En ambos casos, se ilustran las diferencias debidas al efecto de curación en autoclave. Como puede observarse, el efecto "volcán"

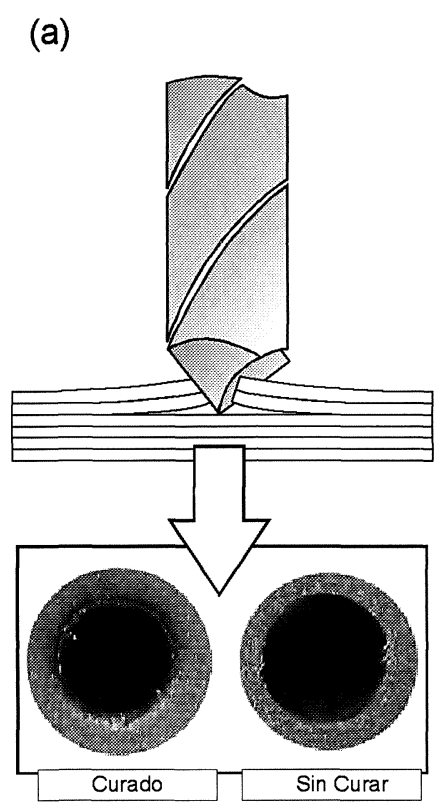

(b)

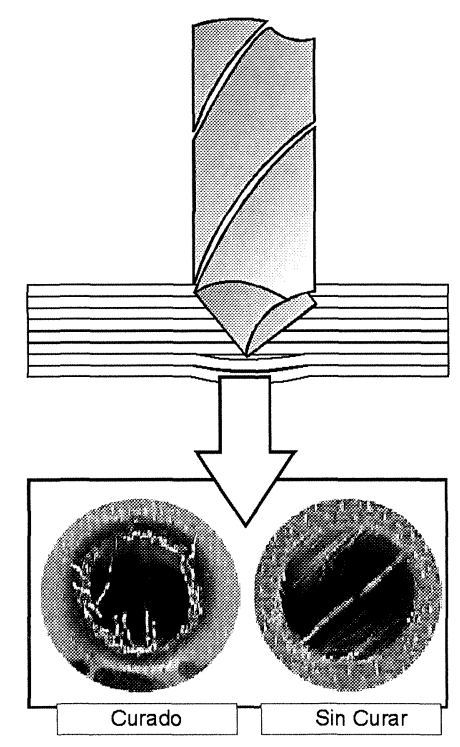

Figura 1. Delaminación de la FC en (a) entrada y (b) salida de la broca.

Figure 1. CF delamination: (a) IN, (b) OUT, of drill. 
de la delaminación se hace mucho más intenso cuando las muestras han sido sometidas a un proceso de curación para darles una mayor cohesión.

Siguiendo la expresión (1), se ha determinado el factor de delaminación para las distintas condiciones de corte aplicadas para cada una de las herramientas utilizadas. En el gráfico de la figura 2 se muestran los resultados obtenidos. Como puede observarse, los mejores resultados se obtienen para herramientas de metal duro con recubrimiento de CrN. No obstante, para velocidades de corte bajas y avances elevados (mínima relación v/a), prácticamente, casi todos los materiales de herramienta han presentado un buen comportamiento.

Sin embargo, estas condiciones no pueden tomarse como las mejores, a pesar de obtenerse una baja delaminación. En efecto, como es bien conocido, el avance introduce una componente en la calidad de acabado superficial de las piezas mecanizadas.

Por otro lado, en el mecanizado de este tipo de materiales, apenas se forma viruta, sino que la fibra es arrancada en pequeñas partículas que, dependiendo de la temperatura, se adhieren a la cara de desprendimiento de la herramienta y a la zona de vaciado de las brocas (Fig. 3a). Este hecho provoca una disminución de la fluencia del material implicando, por una parte, que la acumulación de material cortado en las zonas de evacuación de la herramienta puede conducir al "colapso" del proceso llegando a provocar la rotura de la broca (Fig. 3c). Por otro lado, el material adherido conlleva una pérdida de la geometría ideal de la herramienta, lo cual trae como consecuencia una disminución de calidad de acabado superficial en el interior de los

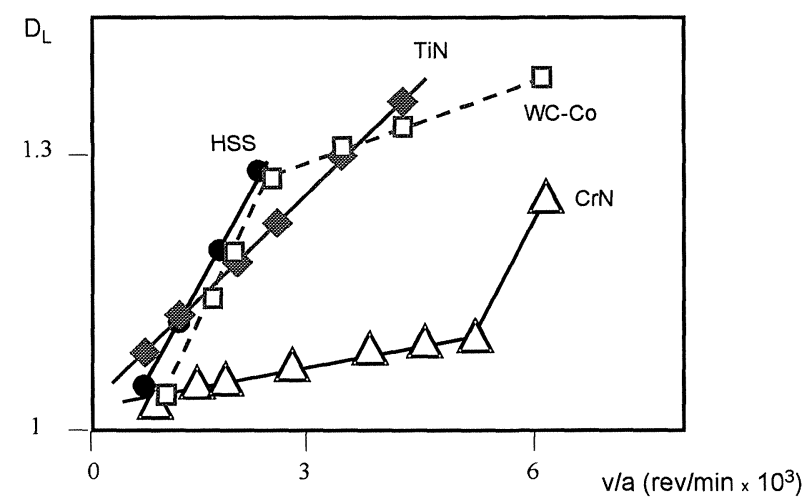

Figura 2. Factor de delaminación en función de la relación $\checkmark /$ a para los distintos materiales de herramienta empleados.

Figure 2. Delamination factor as a function of $v / a$ relation for the different tool materials employed. (a)

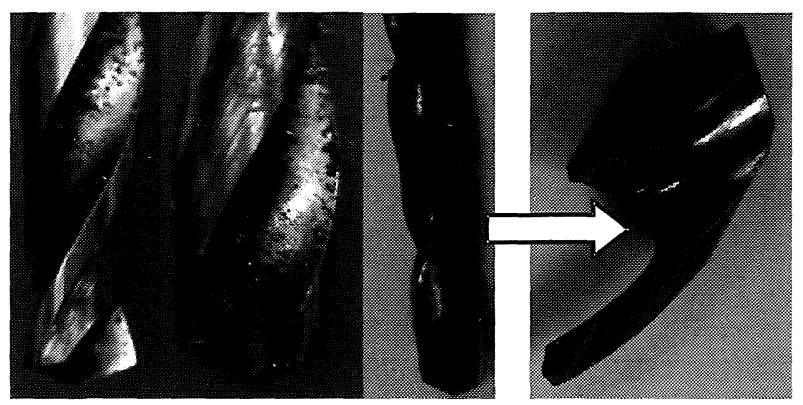

Figura 3. Evolución de la adhesión de partículas de FC a la zona de evacuación de la viruta. (Herramienta: TiN. Relación $v / a=3.350 . N_{T}=92$ ) .

Figure 3. Evolution of adhesion of CF particles to the chip-flow zone of the cutting tool. (Tool: TiN. $v / a=3350 . N_{T}=92$ ).

orificios $^{[10]}$. En la figura 4 se muestra la evolución del parámetro $\mathrm{R}_{\mathrm{a}}$ con el número de taladros para ensayos efectuados con herramientas con recubrimiento TiN en distintas condiciones de corte. Como puede observarse, a medida que disminuye la relación v/a, la calidad superficial disminuye por efecto del aumento del avance, excepto, para el valor mínimo estudiado, en el que puede existir una situación de mejora debido a la velocidad de corte. En la actualidad, se procede a un análisis multivariante en un espectro amplio de velocidades y avances, eliminando la componente natural inducida por este último con objeto de profundizar en la influencia de la velocidad de corte para cada avance.

En cualquier caso, con los datos disponibles, es posible llevar a cabo una primera evaluación del índice de calidad medio para los distintos materiales de herramientas empleados. En el gráfico de la figura 5 se presentan estos índices normalizados al

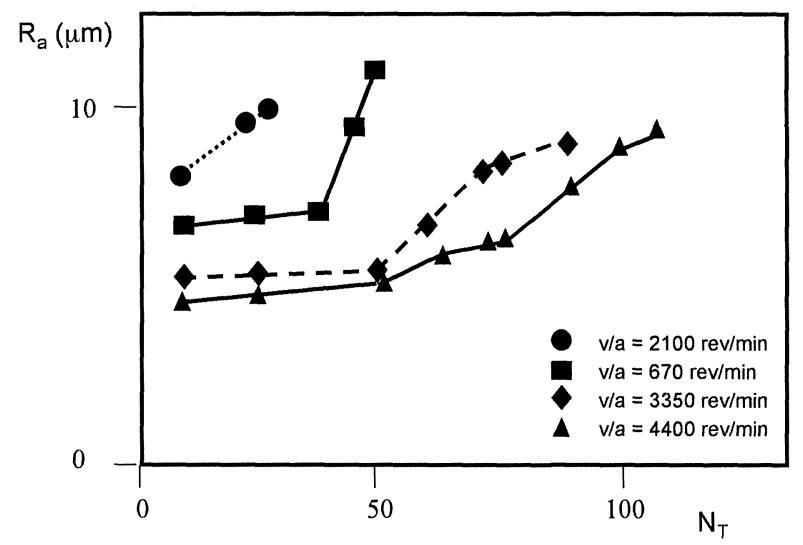

Figura 4. Evolución de $R_{a}$ con el número de taladros para distintas relaciones $v / a$. (Herramienta: TiN).

Figure 4. Evolution of $R_{a}$ as a function of the holes number for different $v / a$ relations. (Tool: TiN). 


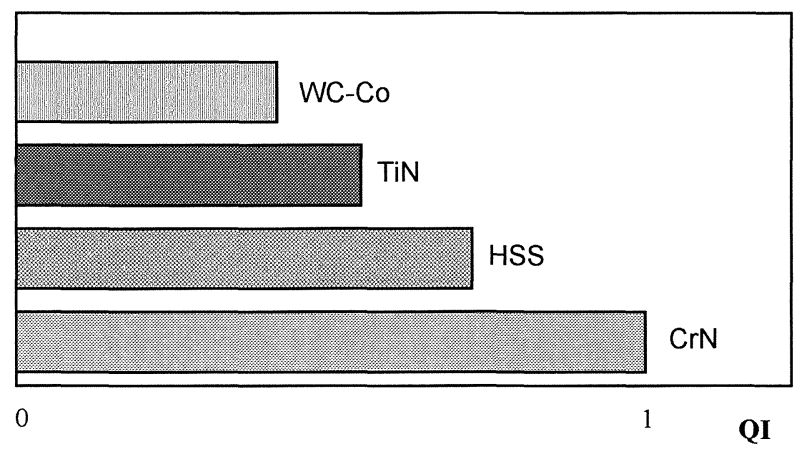

Figura 5. Índice de calidad promediado, normalizado al máximo, para los distintos materiales de herramienta empleados.

Figure 5. Average quality index, normalized to QI maximum, for the different cutting tool materials employed.

valor máximo. Como puede apreciarse, el estudio de los índices de calidad promedio, indican que el material de herramienta más aconsejable para este tipo de mecanizados, en las condiciones de máxima agresividad empleadas y efectuando taladrados a tiempo minimizado (una sola pasada), es el WCCo (metal duro) con recubrimiento de CrN. Para este material, además, los mejores resultados se obtienen para relaciones $\mathrm{v} / \mathrm{a}$ inferiores a 3.000 $\mathrm{rev} / \mathrm{min}$.

\section{CONCLUSIONES}

El taladrado de fibra de carbono conlleva una serie de problemas, tales como delaminaciones, pérdida de calidad en el acabado superficial, desgaste rápido y excesivo de la herramienta de corte o, incluso, rotura catastrófica de la misma.

Se ha analizado el taladrado de planchas de fibra de carbono empleando distintos materiales de herramienta en unos determinados rangos de velocidades de corte y avance. Siguiendo estudios como los presentados en trabajos referidos anteriormente ${ }^{[4 \text { y } 9]}$, se han determinado el factor de delaminación, la calidad superficial y el índice de calidad.

De acuerdo con los resultados obtenidos, a velocidades de corte relativamente bajas y avances moderados o altos, el factor de delaminación no es crítico para ninguno de los materiales empleados. Sin embargo, en estas condiciones se obtiene una rugosidad superficial elevada debido al valor del avance. La compensación de ambos factores se ha introducido en el estudio del índice de calidad. Los resultados obtenidos han mostrado que, para relaciones v/a inferiores a $3.000 \mathrm{rev} / \mathrm{min}$, se obtienen resultados industrialmente aceptables cuando se mecaniza con herramientas de WC-Co recubiertas con $\mathrm{CrN}$.

\section{Agradecimientos}

Este trabajo ha sido financiado por la Comisión Interministerial de Ciencia y Tecnología (CICYT) proyecto DPI2001-3747, por AIRBUS España, S.L. y por la Junta de Andalucía.

\section{REFERENCIAS}

[1] M. Álvarez, M.S. Carrilero, F. Torres, B. Grille, M. Barro y M. Marcos, Proc. XIV Cong. Investigación Máquina-Herramienta y Tecnologías de Fabricación, San Sebastián, 2002, pp. 315-327.

[2] J. Hinrichsen y C. Bautista, Air \& Space Europe 3 (2001) 119-124.

[3] A. Vlot, L.B. Vogelesang y T.J. De VRies, Aircr. Eng. Aerosp. Technol. 71 (1999) 558-570.

[4] Y.A.R APSORO, Master Thesis, Universidad Tecnológica de Delft, Delft, Holanda, 1996.

[5] G.H.J.J. Roebroeks, Fatigue of Aircraft Materials, A. Beukers, Th. De Jong, J. Sinke, A. Vlot y L.B. Vogelesang (Eds.), UPD, Delft, Holanda, 1992.

[6] L.B. Vogelesang, J. Schijve y R. Fredell, Case Studies in Manufacturing with Advanced Materials, A. Demaid y J.H.W. de Wit (Eds.), Elsevier, Amsterdam Holanda, 1995, pp. 74-82.

[7] M.S. Carrilero, M. Álvarez, B. Grille, M. Barro y M. Marcos, VII Reunión Nacional de Materiales, Madrid, 2002, p. 40.

[8] M.S. Carrilero, B. Grille, F. Torres, M. Alvarez y M. MARCOS, I Jomadas Avances en Ingeniería Industrial y Civil, Algeciras, Cádiz, 2002, p. 16.

[9] M.S. Carrilero, B. Grille, J.M. Sánchez, M. Alvarez y M. MARcos, XV Cong. Nac. Ingeniería Mecánica, Libro de Resúmenes, Cádiz, 2002, p. 381.

[10] M.A. Sebastián, J.M. SÁnchez, M.S. Carrilero, J.M. González, M. Álvarez y M. Marcos, Int. J. Manuf. Sci. Prod. 4 (2002) 211-216. 